\title{
Pharmacogenetic testing in primary care practice: opinions of physicians, pharmacists and patients
}

\author{
Marie-Pier Frigon1', Marie-Ève Blackburn², Camélia Dubois-Bouchard², Ann-Lorie Gagnon', \\ Suzie Tardif² \& Karine Tremblay*,1,3 (D) \\ ${ }^{1}$ Community Genomic Medicine Centre \& biocluster ECOGENE-21, Department of Medicine, Université de Montréal, Saguenay, \\ QC, Canada \\ 2ÉCOBES-Research \& Transfer Center, Cégep de Jonquière, QC, Canada \\ ${ }^{3}$ Centre intégré universitaire de santé et de services sociaux du Saguenay-Lac-Saint-Jean, Saguenay, QC, Canada \\ *Author for correspondence: Tel.: +1 4185411000 extension 2193; Fax: +1 418541 7091; karine.tremblay.10@umontreal.ca
}

\begin{abstract}
Aim: Although health authorities have set pharmacogenetic (PGx) markers on labels of hundreds of drugs, the use of PGx in clinical care remains infrequent. The adoption of PGx will depend on the opinions of physicians, pharmacists and patients. Materials \& methods: Qualitative focus group interviews were performed with 23 physicians, 11 pharmacists and 30 patients. Results: Majority of the participants showed enthusiasm toward the implementation of PGx in clinics. Lack of knowledge on PGx, roles of healthcare providers, factors in favor and challenges of PGx implementation, ethical and insurance issues, educational and tools needs were the key themes that emerged from the interviews. Conclusion: Several barriers impede the uptake of PGx in clinics, however, opinions of physicians, pharmacists and patients are mostly favorable.
\end{abstract}

First draft submitted: 8 January 2019; Accepted for publication: 5 April 2019; Published online: 13 June 2019

Keywords: acceptability $\bullet$ implementation $\bullet$ opinions $\bullet$ patients $\bullet$ pharmacists $\bullet$ pharmacogenetics $\bullet$ primary care physicians

Pharmacogenetics (PGx), the study of interactions between genetic factors and drug response, is expected to define tomorrow's medical and pharmaceutical practice in terms of prescriptions, treatment and management of patients [1-3]. PGx testing can help predict a therapeutic response by detecting the presence of variations in one or more genes influencing drug response [4]. PGx can also have a significant implication for patients and clinicians by minimizing the incidence of adverse events according to the genetic variations and reducing the number of medical consultations [5]. In fact, as much as $12 \%$ of emergency visits are due to adverse events caused by a new drug [6]. In the last years, health authorities such as Health Canada and the FDA have set PGx biomarker information on labels of over 100 and 200 drugs, respectively [7]. Despite these recommendations, the use of PGx testing in routine clinical care remains infrequent [8]. Reasons that explain the slow uptake of PGx in clinics include unfamiliarity of health care providers with PGx, cost associated with testing, time constraints, absence of clear clinical guidelines, lack of easily accessible tests and several ethical considerations [2,3,9-14]. Randomized controlled trials (RCT) have provided evidence to support the implementation of PGx-guided therapy [15,16], particularly in the treatment of psychiatric disorders [17-20]. However, RCT are expensive and take a long time before sufficient data are collected to clearly demonstrate the improved clinical outcomes of PGx-guided treatment [21]. Therefore, the clinical utility of PGx testing is still inconclusive [22] and more studies are needed. It is generally well known that primary care providers such as primary care physicians (PCPs) and pharmacists are expected to play an important role in supporting the uptake of PGx into routine clinical care [23]. In fact, more primary care providers are being asked to interpret genetic test results as direct-to-consumer genetic tests are becoming increasingly available [24]. In the last decades, patients have been more involved with their personal healthcare decisions. Their empowerment in the management of PGx data could improve the diffusion of test results among healthcare professionals and thus facilitate the implementation of PGx tests [25]. As PGx implementation will lie on a multidisciplinary framework, 
we postulated that the uptake of PGx in clinics will largely depend on PCPs', pharmacists' and patients' opinions and interests. The specific aim of this study was to better understand the perceptions of PCPs, pharmacists and patients on the implementation of PGx testing in clinical practice. To our knowledge, this is the first qualitative study to consider and compare the opinions of these three groups in the same study. The results of this study may lead to new insights that could inform us on the direction of future implementation efforts.

\section{Materials \& methods}

\section{Study design}

Semi-structured focus group interviews were conducted to elicit the different opinions of PCPs, pharmacists and patients on PGx tests implementation. Focus group interviews were performed as it is the method of choice in the context of an exploratory research in healthcare [26]. Discussion groups are also known to generate a lot of data in a short amount of time [27]. Common agreements and disagreements are rapidly observed during discussion groups making it possible to identify the different opinions and perceptions of the participants [28]. This study adheres to COREQ guidelines for reporting qualitative research [29].

\section{Study settings}

The research team is composed of two co-principal investigators (MEB/KT) with, respectively, a background in sociology and in applied pharmacogenetics, a research coordinator with a master in biomedical sciences (CD), a $\mathrm{PhD}$ student in education (ST) and two MSc students (MPF/ALG). MEB, who conducted the focus groups, is experienced with focus groups and individual interviews [30-32]. CD participated in the focus groups and the data analysis. Graduated students also participated in the data analysis (MPF/ST) and all participated to the writing of the manuscript.

A total of six focus groups (two for each category of participants) were conducted since it is estimated that at least $80 \%$ of themes on a topic are brought up with two to three focus groups by population [33]. PCPs were invited to participate in the study through an announcement made by the director of the regional department of general medicine in Saguenay-Lac-Saint-Jean (SLSJ), QC, Canada. Community pharmacists were also invited to participate through an announcement from the regional department of pharmaceutical services of SLSJ and through the community professional connections using a convenience sampling and snowball recruitment strategies. Patients from the SLSJ general population were recruited through a convenience sampling following public announcement using social media, and leaflets left in pharmacies. Patients were invited to a lunch talk to answer the following question: "What do drugs and genetics have in common?" Participants were asked to choose between two different time slots according to their availability. For reasons of convenience, the interviews were conducted at the hospital for the PCPs' groups and in private rooms in public places (e.g., coffee shops or restaurants) for pharmacists and patients. All participants received a compensation for their time $(\$ 75 \mathrm{CA})$ and a meal was provided during the interviews since they occurred during mealtime.

\section{Data collection}

A semi-directed 60 minute interview guide was used to conduct the discussion groups. The interview guides (see Supplementary File 1) were developed by the co-principal investigator (MEB) and questions were derived from themes reported in her previous work [34] and were adapted to the different groups interviewed. The interview guide was created to be able to reveal the social construction of the meanings and opinions of the different groups interviewed. All interview guides were validated by members of the research team. CD participated as an observer and was responsible to note nonverbal behaviors, group dynamics and any other aspects that were relevant to the research. Topics covered during the interviews were the same for all groups 1) personal and professional experiences; 2) social representation elements regarding genetic and PGx; 3) real-life scenarios using examples of drugs with recommended PGx testing developed by KT; 4) issues surrounding PGx implementation and the specific roles of PCPs and pharmacists in this context; and 5) possible solutions to facilitate the implementation of PGx in frontline practice. Questions addressed were tailored to the different groups interviewed (PCPs, pharmacists and patients). For example, PCPs and pharmacists were asked how PGx could be integrated into their practice while patients were asked which of the two healthcare providers should prescribe PGx tests. The interviews were audio-taped and transcribed verbatim. 
Table 1. Summary of participants' characteristics.

\begin{tabular}{|c|c|c|c|}
\hline Characteristics & Patients $(n=30)$ & Primary care physicians $(n=23)$ & Pharmacists $(n=11)$ \\
\hline Gender (female/male) & $21 / 9$ & $17 / 6$ & $9 / 2$ \\
\hline Mean age (range) & $50.3(19-78)$ & Not asked & Not asked \\
\hline Mean years of practice (range) & Not applicable & $15.0(1-30)$ & $7.5(1-20)$ \\
\hline \multicolumn{4}{|l|}{ Last educational level completed: } \\
\hline - Elementary school & 1 & & \\
\hline - High school & 14 & & \\
\hline - College & 8 & & \\
\hline - Bachelor's degree & 4 & & \\
\hline - Master's degree & 3 & & \\
\hline
\end{tabular}

\section{Data analysis}

NVivo (version 11, QSR International Pty, Ltd), an inductive thematic software, was used to analyze the verbatim. A content analysis, inspired by the thematic analysis model proposed by Paillé and Mucchielli [35] was conducted independently by ST and CD. The preliminary coding frame was developed using different themes derived from the interview guide and codes were refined throughout the analysis to include emerging themes. Discourse analysis was performed on the concrete examples on PGx applications and gave a better understanding of the participants' opinions of PGx. A vertical analysis of the data was first conducted to understand their general opinion for each topic discussed during the interviews and a transverse analysis was then performed to explore the recurrent themes and the differences between the three groups [36]. Any discrepancies were discussed between ST and CD and a third researcher $(\mathrm{MEB})$ to ensure triangulation [37].

\section{Ethical considerations}

This study was approved by the Cégep de Jonquière Institutional Ethic Review Board (CER2-11-2016-02). Authors declare no conflict of interest.

\section{Results}

\section{Participants' characteristics}

In total, 23 PCPs (group $1=11$; group $2=12$ ), 11 pharmacists (group $1=7$; group $2=4$ ) and 30 patients (group 1 = 15; group $2=15$ ) participated in one of the six focus groups conducted between November 2016 and April 2017. Participants' characteristics are summarized in Table 1 . Following analyses of the interviews verbatim, seven key themes emerged from the data (described in next subsections) .

\section{Awareness on PGx}

Questions to see what the participants knew about PGx were first asked. Healthcare professionals interviewed had a generally broad idea of the definition of PGx. Most of them knew about it but had limited clinical experience. When asked by the interviewers, "When I tell you about PGx-guided therapy, what do you think of first?" all four groups of PCPs and pharmacists referred to PGx's use in cancer therapy. Three of the four groups mentioned the lack of awareness on PGx in clinical practice. One of the participants mentioned: "It's something that is just around the corner, but we don't know at all," (PCP, group 1). When the interviewers explained to the groups of patients the concept of PGx, they had many opinions about it, demonstrating their interest with this science. One of them shared this thought: "I am convinced that there is a link between genetics and drug treatment. Although historically, trains of thought were such that if an individual was not responding to a treatment, then it meant that the individual was having somatic symptoms. That was the term we were using. I am happy that you are bringing this up because this might avoid people being told that they are having somatic symptoms," (Patient, group 2), while another patient mentioned, "I think, I am pretty sure in fact, that the link [for drug response] is genetic, but like many people, I can't explain why," (Patient, group 2).

\section{Roles of healthcare providers in PGx implementation}

All six groups were asked to give their opinions on the roles PCPs and pharmacists should play in the implementation of PGx testing. All four groups of health care professionals mentioned that pharmacists should play a major role in 
the implementation of those tests in clinical practice. Some participants suggested that pharmacists could review prescriptions made by the PCPs and then suggest a modification to a treatment if a PGx test is recommended. One of the PCPs described how pharmacists could be involved in PGx testing: "I would not do this for sure, I would not have time, I wouldn't be able. [... ] I would talk to the pharmacist about it. Ifyou [pharmacists] know there is something wrong with one cytochrome, you could warn me if I prescribe something related to that." (PCP, group 2).

The idea that pharmacists could prescribe a PGx test and choose the drug according to the results of the test was also mentioned by the pharmacists' groups: "Our role is to follow the patient, to make sure our treatment is effective and well tolerated. So, to prescribe a [pharmaco] genetic test to prove it is effective [...] that's what we do," (Pharmacist, group 2) as well as the PCPs' groups: "Maybe this could be a way of doing things; the physician makes the diagnosis and the pharmacist chooses the drug," (PCP, group 1).

Surprisingly, patients were more reluctant to the idea of having pharmacists prescribing PGx tests and choosing the drugs. One of them mentioned: "They are the drug sellers, they lost me already" (Patient, group 1). Another patient specified "[The pharmacist] could give me a paper and I would pass a blood test. He would receive the result [... ] contact my physician to see if a dosage modification is needed, if it needs to be increased, but he wouldn't necessarily be the one who makes the decision," (Patient, group 2).

Factors in favor of the implementation of $P G x$

All groups were asked what factors could facilitate the uptake of PGx into clinical practice. Unanimously, all groups mentioned the reduction of adverse events as being one of the main benefits of PGx testing: "All drugs that were given to me, going to the hospital, having adverse effects, drug rejection [.. ], I could have had fewer adverse effects, fewer visits paid to the emergency room and my quality of life could have been better," (Patient, group 2). Moreover, clinicians stated that PGx could enable them to spend more time on other aspects of their practice by saving them time on dealing with drugs' adverse events.

Most clinicians agreed on the fact that the results of PGx tests had to be rapidly obtained to be valuable. As one of the PCPs mentioned: "If the tests existed and were available at a reasonable cost with above all, a rapid response, we would apply them for sure," (PCP, group 1). Some patients stated that they would prefer not to wait for the results of the tests before starting the medication. They preferred being prescribed a smaller dose of the drug while waiting for the results of the test and adjusting it accordingly.

\section{Challenges in the implementation of $P G x$}

One of the themes covered during the interviews concerned the challenges associated with PGx implementation. Cost of PGx testing was seen as the greatest reluctance to its implementation for most of the participants interviewed. In fact, participants highlighted the fact that the decision related to the cost of those tests is one that needs to be taken by the society, as specified by a pharmacist: "Who will pay for this? The government? Will it be covered? Will the taxpayers agree with that?" (Pharmacist, group 1). Interestingly, patients seemed to be more inclined to pay for those tests. One of them said: "We have a financial concern but, above all, we want to be healthy," (Patient, group 2).

Most groups of healthcare professionals mentioned that accessible PGx guidelines were needed before PGx testing could be used efficiently: "[...] there are some studies, but we never heard about those. We never read them, nobody ever told us about them. Honestly, we're waiting for a guideline or a defined clinical approach," (PCP, group 2).

\section{Ethical \& insurance issues}

PCPs and pharmacists were asked during the interviews: "What should patients know before doing the test?" Both stated that the objectives, implications and consequences of the tests should be clearly explained to the patients before they take it: "This isn't simply a blood test," (Pharmacist, group 2), "We need to explain to them what changes it's going to make in their life," (PCP, group 2).

Throughout the group discussion, some participants mentioned that revealing genetic information could cause anxiety to the patients: "According to the test, it becomes negative for him [the patient] to know that he carries a gene, but that he may never suffer from the disease. It can make him anxious [...]" (PCP, group 2). When we asked the patients what they think they should know before doing the test, some patients believed they should not know everything about their DNA: "It's better not to know about it [genetic information]," (Patient, group 1). Health professionals highlighted the fact that, since PGx implies taking a genetic test, the patient's family was inherently going to be concerned by the results of the test: "Because if he [the patient] receives a genetic test positive for slow metabolizer, then maybe some family members are slow metabolizers as well," (PCP, group 1). 
Other ethical issues were also brought up in the discussion groups. A large majority of patients were mainly concerned about the confidentiality of the results: "We don't want everybody to know about it [results of the test], but we want professionals to know about it," (Patient, group 2). Concerns about insurance companies having access to PGx results were also mentioned by all groups interviewed: "The insurance company will not be willing to pay anymore. You won't be insured for depression because you are a slow metabolizer," (PCP, group 2).

When addressing the question of the cost of PGx testing, patients agreed on the fact that there should be no social inequalities with the venue of those tests: "We would not accept a medicine for the rich and one for the poor for the DNA test," (Patient, group 2). They advocated for the tests to be accessible to everyone, like most of the public healthcare in Quebec.

\section{Training \& educational needs}

PCPs and pharmacists were asked their opinion about the needs for training in PGx. Most groups stated that implementation of PGx in clinical practice should come with an appropriate training for health professionals. Moreover, there was a general consensus among the groups toward the lack of training in PGx in medical and pharmacy programs and as part of continuing education: "I will for sure want training to know what it is about and to know the pros and the cons," (PCP, group 2).

\section{Tools needed}

Participants were also asked during the focus groups what tools they would like to have in support of PGx implementation. During the interviews, it was found that medical computerization was still a slow process in Quebec's healthcare system. All professionals agreed on the fact that, in order to be implemented correctly, PGx data should be integrated into the software programs used by health professionals. In fact, one of the pharmacists shared this thought: "It would be essential to have it included into a software that can help us not to miss some information," (Pharmacist, group 2). The idea of integrating electronic alerts was suggested by one of the PCPs: "The prescriber should be aware that he [the patient] is a slow metabolizer and that when we prescribe this, it makes BEEP [alarm sound], " (PCP, group 1). Most patients also agreed that results of those tests should be computerized and put into a universal electronic medical record (EMR), therefore all information could be easily accessible to any professionals. Patients also mentioned that tools to facilitate their comprehension of PGx such as information pamphlets, reliable websites, phone lines and books would be of great help.

\section{Discussion \& conclusion}

This study explored PCPs', pharmacists' and patients' opinions on the imminent uptake of PGx testing in routine clinical care. A general enthusiasm toward the future implementation of PGx in clinics was perceived among the six groups interviewed. The findings from this study raised a lack of awareness on PGx among the health professionals interviewed. In fact, PCPs and pharmacists participating in the present study mostly stated they had very little knowledge on PGx outside its application to cancer therapy.

The unfamiliarity of healthcare providers with PGx has been addressed in multiple previous studies [23,38-43]. For example, De Denus and Colleagues conducted a survey among Quebec's (Canada) pharmacists. Of the 284 pharmacists surveyed, only $7.7 \%$ considered themselves as being comfortable receiving and handling PGx test results [39]. Similarly, a nationwide survey conducted among US PCPs and specialists revealed that only $10.3 \%$ of them felt they had adequate knowledge on PGx testing availability and applicability [43]. In the present study, none of the PCPs and pharmacists interviewed had been using PGx in their daily practice. This contrasts with findings from Haga and Colleagues [42], who found that $20 \%$ of the PCPs interviewed in the USA had ordered at least one PGx test per year. Our results might be explained by the fact that, at the time this study was conducted, PGx testing in Canada was either offered by private companies or by some institutions as part of a research project [44].

Given the ever-increasing field of genomics and the lack of increase in the number of genetic professionals, it is expected that frontline healthcare providers will be responsible for the integration of PGx into routine clinical care over the next years $[24,45]$. No clear consensus has been made on which health professionals should act as main actors in PGx implementation. Some authors believe that pharmacists are better prepared in terms of their competencies to support the implementation of PGx testing [38,44]. While Peterson and Colleagues found no agreement among clinicians on who should be delivering PGx tests and results [46], PCPs and pharmacists surveyed in other studies mentioned that both professionals should be playing a role and should be working in close collaboration [39,47]. These findings are similar to what was found in the present study. In fact, when asked whether the PCPs or 
the pharmacists should be in charge of PGx testing, no clear consensus was observed between the two groups of healthcare professionals, yet it was reiterated that both of them should be involved. Interestingly, patients mostly believed that PCPs, not pharmacists, should be in charge of PGx testing. This corroborates with findings from Payne and Colleagues who found that neither physicians nor patients agreed that pharmacists should be responsible for explaining PGx test results [48]. Both PCPs and patients interviewed in this study mentioned that pharmacists were inherently implicated in the drug dispensary process, making them at risk of having a certain degree of conflict of interest, which could explain this finding.

PGx well-known premises such as decrease in the number of adverse events and improved efficacy of the drugs have been addressed as main benefits of PGx by most of the participants in the present study. Similar findings have been found in other studies $[41,49,50]$. Owusu Obeng and colleagues, who performed a survey among physicians applying PGx, found that $77 \%$ of physicians believed PGx testing results gave them valuable information [23]. On the other hand, numerous challenges surrounding the implementation of PGx have been mentioned by the participants in the present study. Although the cost of PGx tests was perceived as a main barrier to the implementation of PGx by the participants in this study and many others [38,50,51], it is no longer considered as such. In fact, the development of multigene PGx tests considerably reduced the cost of PGx testing [21]. PCPs and pharmacists interviewed in this study also mentioned the lack of clinical guidelines as being an obstacle to the adoption of PGx. Yet clinical guidelines supporting the development of PGx have been developed by some institutions such as the Clinical Pharmacogenetics Implementation Consortium [52]. This finding might suggest that current clinical guidelines are not appropriately communicated to frontline healthcare providers, such as PCPs and community pharmacists. Easily accessible clinical guidelines should be provided to healthcare providers with details on the various steps of PGx test prescription (when to prescribe a PGx test, how to do it, where to send the samples and how to manage PGx test results).

Ethical issues such as insurance coverage, accessibility and data protection (confidentiality) were highlighted by all groups of participants. Such concerns have also been iterated in the literature [39,43,53,54]. To address these issues, laws and regulations on data protection have been issued in North America during the past years [55]. In Canada, the Genetic Non-Discrimination Act has been in effect since 2017 [56]. However, the existence of this law seems to be unknown to healthcare professionals and patients as none of them referred to it during the interviews. Clear and concrete regulations surrounding genetic data protection should be issued by authorities and publicized.

Consistent with other studies assessing the barriers and facilitators of PGx implementation [23,24,39,42,51], the lack of genomic education in medical and pharmacy programs and the lack of continuing education on PGx was addressed by the participants in this study. When performing a survey on medical and pharmacy students, Yau and Haque found that over 90\% of pharmacy students have had a university class on PGx compared with 57\% of medical students [57]. This finding suggests that pharmacy graduates may be better prepared than PCPs for the arrival of PGx in clinics. Changes at the level of medicine and pharmacy curricula will be critical to ensure a proper PGx' training of future PCPs and pharmacists [58]. On the other hand, results from a recent study have shown that physicians with five or fewer years of practice were more comfortable and more confident dealing with PGx results [23]. Continuing education on PGx for physicians and pharmacists in practice will be needed to ensure that these professionals are fully prepared to adopt PGx testing. A personalized PGx training, tailored to the healthcare professionals, their setting and context should be offered [58]. In this regard, Genome Quebec and the Quebec Network for Personalized Health Care recently launched an online training for health professionals interested in learning more about genomics and personalized medicine [59] paving the way to the implementation of genomics in primary care. In order to appropriately support the uptake of PGx testing in clinics, this online training should include clear and valuable information on PGx and should be mandatory for all healthcare providers that will have to deal with PGx over the next years.

All groups participating in this study discussed the need for tools that could facilitate the implementation of PGx. While patients were more concerned with having a universal EMR, physicians and pharmacists emphasized on the need to incorporate PGx information into existing software programs. Clinical decision support (CDS) systems are used to deliver PGx recommendations based on existing guidelines and can be integrated into the EMR. Several PGx implementation programs have developed CDS [60] and some include CDS alerts, which give the clinicians specific, relevant and actionable information that facilitate the adoption of PGx by the users [61]. An important project on the implementation of PGx testing using a decision support tool, The Ubiquitous Pharmacogenomics (U-PGx), is currently being conducted in seven different countries across Europe [62]. This study is using a CDS 
system that can either be integrated to the EMR or outside as a pocket card. This study and others are warranted in order to demonstrate the clinical utility of such CDS.

The results reported here provide information on PCPs', pharmacists' and patients' opinions on the implementation of PGx into routine clinical care provided in the Quebec Health System.

Some limitations should be acknowledged. First, some minor themes might not have been covered by the participants during our focus groups since up to $20 \%$ of themes may still be uncaptured by two focus groups [33]. However, to ensure the reliability of our results, three different data sources (PCPs, pharmacists and patients) and two focus groups per population were performed, which allowed the triangulation of data. The data obtained enabled us to compare the different themes covered across the studied groups and to come up with potential solutions to facilitate the implementation of PGx in clinics. We also have been able to replicate the main reported themes found in the literature. Second, since the participants were all volunteers who were selected according to a convenience sampling strategy, they may not be representative of the population and thus, the results of this study may not be generalizable to the whole population of PCPs, pharmacists and patients.

In conclusion, this study, in addition to giving the opinions of PCPs, pharmacists and patients on PGx testing, allowed us to verify the openness of these community stakeholders to host PGx in their clinical practice. For it to be possible, education and close ground collaborations will be needed and should consider the environment and cultural particularities. A general acceptability on the implementation of PGx testing was shown by this study, but its clinical utility will need to be well established and described in guidelines. The current gap preventing PGx testing to be implemented relies on inefficient communication channels. Efforts in creating a communication network allowing these healthcare professionals and other stakeholders to collaborate will be essential. Such harmonized work taking into account clinical environment and socio-organizational specificities needs to be developed.

Executive summary

- Primary care physicians, pharmacists and patients all showed a great enthusiasm toward the entry of pharmacogenetics (PGx) in primary care.

- Costs associated with PGx testing, ethical issues and lack of clinical guidelines are the main barriers to the implementation of PGx perceived by primary care physicians, pharmacists and patients.

- Healthcare professionals lack of awareness on PGx. Educational resources for medical and pharmacy students as well as continuing education for healthcare professionals in practice are needed to facilitate the implementation of PGx in clinical care.

- The roles of primary care physicians and pharmacists in the implementation of PGx need to be clearly defined.

- Close collaboration among all healthcare professionals is essential.

- Clinical utility of PGx testing will need to be demonstrated by further studies and shared with healthcare professionals.

Supplementary data

To view the supplementary data that accompany this paper please visit the journal website at: https://www.futuremedicine.com/d oi/suppl/10.2217/pgs-2019-0004

\section{Author contributions}

M-P Frigon has performed NVivo analyses and their interpretation and has written the manuscript. ME Blackburn has coconceived the study, supervised all focus group interviews and analyses, revised and approved the manuscript final version. C Dubois-Bouchard has planned and coordinated the participants' recruitment, participated to the focus group interviews, performed the NVivo analyses and revised the manuscript. A-L Gagnon has participated to the manuscript handling, interpretation of the results and revised all versions. S Tardif has performed analyses validation and revised manuscript. K Tremblay has coconceived the study, participated to focus group interviews and interpretation of the results, revised all manuscript versions and approved the final version to be submitted.

\section{Acknowledgments}

We would like to thank Charles Caron, for his help with verbatim transcription and all participants who took part in this study. We also want to address a special thank to our respective research center that made possible the realization of this study. Authors MPF and ALG were supported by a scholarship grant from biocluster ECOGENE-21, a non-for-profit research organization. 
Financial \& competing interests disclosure

This research was funded by the Social Sciences and Humanities Research Council of Canada (SSHRCC) \# 430-2016-01243. The authors have no other relevant affiliations or financial involvement with any organization or entity with a financial interest in or financial conflict with the subject matter or materials discussed in the manuscript apart from those disclosed.

No writing assistance was utilized in the production of this manuscript.

Ethical conduct of research

The authors have obtained approval from the Cégep de Jonquière Institutional Ethic Review Board (CER2-11-2016-02). Informed written consent has been obtained from all participants involved in this study.

\section{References}

Papers of special note have been highlighted as: $\bullet$ of interest; $\bullet \bullet$ of considerable interest

1. Green JS, O'Brien TJ, Chiappinelli VA, Harralson AF. Pharmacogenomics instruction in US and Canadian medical schools: implications for personalized medicine. Pharmacogenomics 11(9), 1331-1340 (2010).

2. Dion-Labrie M, Fortin MC, Hébert MJ, Doucet H. Réflexion éthique sur la médecine personnalisée: l'alliance de la science et de la médecine enfin réalisée? Rev. Colomb. Bioét. 3(2), 33-56 (2008).

3. Commission De L'éthique En Science Et En Technologie (CEST). Soins de santé personnalisés: document de consultation. QC, Canada (2012). www.ethique.gouv.qc.ca/fr/assets/documents/SSP/SSP_document_de_consultation_Web.pdf

4. Spear BB, Heath-Chiozzi M, Huff J. Clinical application of pharmacogenetics. Trends Mol. Med. 7(5), 201-204 (2001).

5. Lee JW, Aminkeng F, Bhavsar AP et al. The emerging era of pharmacogenomics: current successes, future potential, and challenges. Clin. Genet. 86(1), 21-28 (2014).

6. Zed PJ, Abu-Laban RB, Balen RM et al. Incidence, severity and preventability of medication-related visits to the emergency department: a prospective study. CMAJ 178(12), 1563-1569 (2008).

7. PharmGKB. Drug label annotations (2018). www.pharmgkb.org/labelAnnotations

8. Singh A. Pharmacogenomics: the potential of genetically guided prescribing. Austr. Fam. Phys. 36(10), 820-824 (2008).

9. Letarte N, Lavoie A, Sheehan N et al. La pharmacothérapie personnalisée et la pharmacogénétique. Pharmactuel 46(4), 258-268 (2013).

10. Ndegwa S. La pharmacogénomique et la warfarine [Notes sur les technologies de la santé en émergence]. Agence Canadienne des Médicaments et des technologies de la santé 104, 1-8 (2007).

11. Poncin J. Les Américains sont-ils favorables à la pharmacogénomique? L'Observatoire de la génétique (2003). www.omics-ethics.org/observatoire/zoom/zoom_03/z_no10_03/z_no10_03_2.html

12. Gossard F, Hamet P. Pour distinguer génétique, génomique, pharmacogénétique et pharmacogénomique dans les problèmes éthiques. Les pratiques de recherche biomdicales visitées par la bioéthique (2003).

13. Hall SS. Personalized medicine's bitter pill. MIT Technology Review ( 2003). www.technologyreview.com/s/401777/personalized-medicines-bitter-pill/

14. Rothstein MA. Pharmcogenomics Social, Ethical and Clinical Dimension. Éditions John Wiley \& Sons (2003).

15. Mallal S, Phillips E, Carosi G et al. HLA-B*5701 screening for hypersensitivity to abacavir. N. Engl. J. Med. 358(6), 568-579 (2008).

16. Pirmohamed M, Burnside G, Eriksson N et al. A randomized trial of genotype-guided dosing of warfarin. N. Engl. J. Med. 369(24), 2294-2303 (2013).

17. Olson MC, Maciel A, Gariepy JF et al. Clinical impact of pharmacogenetic-guided treatment for patients exhibiting neuropsychiatric disorders: a randomized controlled trial. Prim Care Companion CNS Disord 19(2), doi: 10.4088/PCC.16m02036. (2017).

18. Bradley $\mathrm{P}$, Shiekh $\mathrm{M}, \mathrm{Mehra} \mathrm{V}$ et al. Improved efficacy with targeted pharmacogenetic-guided treatment of patients with depression and anxiety: a randomized clinical trial demonstrating clinical utility. J Psychiatr Res 96, 100-107 (2018).

19. Perez V, Salavert A, Espadaler J et al. Efficacy of prospective pharmacogenetic testing in the treatment of major depressive disorder: results of a randomized, double-blind clinical trial. BMC Psychiatry 17(1), 250 (2017).

20. Ielmini M, Poloni N, Caselli I et al. The utility of pharmacogenetic testing to support the treatment of bipolar disorder. Pharmgenomics Pers Med 11, 35-42 (2018).

21. Wiltshire T, Dong OM. Clinical pharmacogenetics: how do we ensure a favorable future for patients? Pharmacogenomics 19(6), 553-562 (2018)

22. Drozda K, Pacanowski MA. Clinical trial designs to support clinical utility of pharmacogenomic testing. Pharmacotherapy 37(9), 1000-1004 (2017).

23. Owusu Obeng A, Fei K, Levy KD et al. Physician-reported benefits and barriers to clinical implementation of genomic medicine: a multi-site IGNITE-network survey. J. Pers. Med. 8(3), pii:E24 (2018). 
-• This survey assesses the perceived barriers and facilitators of pharmacogenetic implementation among clinicians working in implementation clinics.

24. Selkirk CG, Weissman SM, Anderson A, Hulick PJ. Physicians' preparedness for integration of genomic and pharmacogenetic testing into practice within a major healthcare system. Genet. Test. Mol. Biomarkers 17(3), 219-225 (2013).

25. Hull LE, Lehmann LS, Lynch JA. Gene-based prescribing is here - are providers ready? Am. J. Med. pii: S0002-9343(19)30231-1 (2019). (Epub ahead of print).

26. Birch L, Pétry F. L'utilisation des groupes de discussion dans l'élaboration des politiques en santé. Recherches qualitatives 29(3), 103-132 (2011).

27. Rabiee F. Focus-group interview and data analysis. Proc. Nutr. Soc. 63(4), 655-660 (2004).

28. Baribeau C, Germain M. L'entretien de groupe: considérations théoriques et méthodologiques. Recherches Qualitatives 29(1), 28-49 (2010).

- Provides the methodological approach to conduct focus group interviews.

29. Tong A, Sainsbury P, Craig J. Consolidated criteria for reporting qualitative research (COREQ): a 32-item checklist for interviews and focus groups. Int J Qual Health Care 19(6), 349-357 (2007).

30. Blackburn MĖ, Gaudreault M, Tremblay J-F, Veillette S. Portrait des 16-24 ans sans diplôme résidant sur le territoire de la Commission scolaire de Charlevoix. 93 (2005).

31. Blackburn M-Ė, Joyal I, Brooks S, Tardif S, Arbour N. Étude sur l'abandon et la persévérance à la Mesure de formation de la main-d'œuvre d'Emploi-Québec. 98 (2013).

32. Blackburn M-Ė, Tardif S. Étude sur l'abandon et la persévérance de trois mesures d'aide à l'emploi d'Emploi-Québec. 61 (2016).

33. Guest G, Namey E, McKenna K. How many focus groups are enough? Building an evidence base for nonprobability sample sizes. Field Methods 29(1), 3-22 (2017).

34. Blackburn M-Ė. Les représentations sociales de la pharmacogénomique au Québec: éléments de prospective. Thèse de doctorat en sciences humaines appliquées 415 (2012).

35. Paillé P, Mucchielli A. L'analyse qualitative en sciences humaines et sociales. (4th). Colin A (Ed.). Paris, France (2016).

36. Miles MB, Huberman AM. Qualitative data analysis: An expanded sourcebook, 2nd ed. Sage Publications, Inc, CA, USA (1994).

37. Denzin NK. Interpretive Interactionism. 2, Sage Publications, Inc, CA, USA (2001).

38. Chan CY, Chua BY, Subramaniam M, Suen EL, Lee J. Clinicians' perceptions of pharmacogenomics use in psychiatry. Pharmacogenomics 18(6), 531-538 (2017).

39. De Denus S, Letarte N, Hurlimann T et al. An evaluation of pharmacists' expectations towards pharmacogenomics. Pharmacogenomics 14(2), 165-175 (2013).

-• Surveys Quebec's pharmacists on their opinions ans concerns about pharmacogenomics' implementation.

40. Mccullough KB, Formea CM, Berg KD et al. Assessment of the pharmacogenomics educational needs of pharmacists. Am. J. Pharm. Educ. 75(3), 51 (2011).

41. Fargher EA, Eddy C, Newman W et al. Patients' and healthcare professionals' views on pharmacogenetic testing and its future delivery in the NHS. Pharmacogenomics 8(11), 1511-1519 (2007).

42. Haga SB, Burke W, Ginsburg GS, Mills R, Agans R. Primary care physicians' knowledge of and experience with pharmacogenetic testing. Clin. Genet. 82(4), 388-394 (2012).

43. Stanek EJ, Sanders CL, Taber KA et al. Adoption of pharmacogenomic testing by US physicians: results of a nationwide survey. Clin. Pharmacol. Ther. 91(3), 450-458 (2012).

- This national survey performed among US physicians assess factors influencing pharmacogenomics adoption.

44. Cohn I, Cohn RD, Ito S. Professional opportunity for pharmacists to integrate pharmacogenomics in medication therapy. Can. Pharm. J. (Ott) 151(3), 167-169 (2018).

45. Scheuner MT, Sieverding P, Shekelle PG. Delivery of genomic medicine for common chronic adult diseases: a systematic review. JAMA 299(11), 1320-1334 (2008).

46. Peterson JF, Field JR, Shi Y et al. Attitudes of clinicians following large-scale pharmacogenomics implementation. Pharmacogenomics J. 16(4), 393-398 (2016)

47. Haga SB, Mills R, Moaddeb J, Lapointe NA, Cho A, Ginsburg GS. Primary care providers' use of pharmacist support for delivery of pharmacogenetic testing. Pharmacogenomics 18(4), 359-367 (2017).

48. Payne K, Fargher EA, Roberts SA et al. Valuing pharmacogenetic testing services: a comparison of patients' and health care professionals' preferences. Value Health 14(1), 121-134 (2011).

49. Rogausch A, Prause D, Schallenberg A, Brockmoller J, Himmel W. Patients' and physicians' perspectives on pharmacogenetic testing. Pharmacogenomics 7(1), 49-59 (2006). 
50. Lemke AA, Hutten Selkirk CG, Glaser NS et al. Primary care physician experiences with integrated pharmacogenomic testing in a community health system. Per. Med. 14(5), 389-400 (2017).

- This qualitative study explored the opinions of primary care physicians on the values and challenges of clinical pharmacogenomics.

51. Albassam A, Alshammari S, Ouda G, Koshy S, Awad A. Knowledge, perceptions and confidence of physicians and pharmacists towards pharmacogenetics practice in Kuwait. PLoS One 13(9), (2018).

52. Relling MV, Klein TE. CPIC: Clinical Pharmacogenetics Implementation Consortium of the Pharmacogenomics Research Network. Clin. Pharmacol. Ther. 89(3), 464-467 (2011).

53. Mcmahon T, Tucci J. The perceptions of pharmacists in Victoria, Australia on pharmacogenetics and its implications. Pharm. Pract. (Granada) 9(3), 141-147 (2011).

54. Tuteja S, Haynes K, Zayac C, Sprague JE, Bernhardt B, Pyeritz R. Community pharmacists' attitudes towards clinical utility and ethical implications of pharmacogenetic testing. Per Med 10(8), 793-800 (2013).

55. Prince AE, Roche MI. Genetic information, non-discrimination, and privacy protections in genetic counseling practice. J. Genet. Couns. 23(6), 891-902 (2014).

56. Genetic Non-Discrimination Act Canadian Minister of Justice (2018).

57. Yau A, Haque M. Pharmacogenomics: knowledge, attitude and practice among future doctors and pharmacists - a pilot study. J. Appl. Pharm. Sci. 6(2), 141-145 (2016).

- This study reveals the lack of education on pharmacogenomics at the level of university.

58. Lesko LJ, Johnson JA. Academia at the crossroads: education and training in pharmacogenomics. Per. Med. 9(5), 497-506 (2012).

59. Génome Québec, Inc. Introduction à la génomique, QC, Canada (2018). www.genomequebec-education-formations.com/formations.

60. Mukerjee G, Huston A, Kabakchiev B, Piquette-Miller M, Van Schaik R, Dorfman R. User considerations in assessing pharmacogenomic tests and their clinical support tools. NPJ Genom. Med. 3(26), (2018).

61. Devine EB, Lee CJ, Overby CL et al. Usability evaluation of pharmacogenomics clinical decision support aids and clinical knowledge resources in a computerized provider order entry system: a mixed methods approach. Int. J. Med. Inform. 83(7), 473-483 (2014).

62. Blagec K, Koopmann R, Crommentuijn-Van Rhenen $\mathrm{M}$ et al. Implementing pharmacogenomics decision support across seven European countries: the ubiquitous pharmacogenomics (U-PGx) project. J. Am. Med. Inform. Assoc. 25(7), 893-898 (2018). 